\title{
Preparation interval and cue-utilization in the prevention of distraction
}

\author{
János Horváth
}

Institute of Cognitive Neuroscience and Psychology, Research Centre for Natural Sciences, Hungarian Academy of Sciences, Budapest, Hungary

\section{Address correspondence to:}

János Horváth

Institute of Cognitive Neuroscience and Psychology

RCNS, Hungarian Academy of Sciences

P.O.B. 398, Szondi u 83/85

H-1394 Budapest

HUNGARY

Phone: +36 13542290

Fax: +36 13542416

E-mail: horvath.janos@ttk.mta.hu 


\begin{abstract}
Maintaining a selective attention set allows us to efficiently perform sensory tasks despite the multitude of concurrent sensory stimuli. Unpredictably occurring, rare events nonetheless capture our attention, that is, we get distracted. The present study investigated the efficiency of control over distraction as a function of preparation time available before a forthcoming distracter. A random sequence of short and long tones (100 or $200 \mathrm{~ms}$ with 50$50 \%$ probability) was presented. Independently from tone duration, occasionally $(13.3 \%$ of the time), the pitch of a tone was changed. Such rare pitch variants (distracters) usually lead to delayed and less precise discrimination responses, and trigger a characteristic series of eventrelated potentials (ERPs) reflecting the stages of distraction-related processing: starting with negative ERPs signaling the sensory registration of the distracter; a P3a - usually interpreted as a reflection of involuntary attention change; and finally the so-called reorienting negativity signaling the restoration of the task-optimal attention set. In separate conditions, 663 or 346 ms before each tone (long or short cue-tone interval) a visual cue was presented, which signaled whether the forthcoming tone was a distracter (rare pitch variant), with $80 \%$ validity. As reflected by reduced reaction time delays and P3a amplitudes, valid cues led to the prevention of distraction, but only in the long cue-tone interval condition. The analyses of the cue-related P3b and contingent negative variation showed that participants made more effort to utilize cue-information to prevent distraction in the long cue-tone-, than in the short cuetone interval condition.
\end{abstract}

Keywords: attention, distraction, cognitive control, prediction, event-related potentials (ERPs), P3a 


\section{Introduction}

Efficient goal-directed behavior often depends on our ability to prepare for the occurrence of a set of goal-relevant sensory events. Maintaining such an attention set makes it possible to process task-relevant sensory events as soon as possible when they occur, while allowing us to disregard task-irrelevant events. A "perfect" attention set, however, cannot be achieved: unpredictably occurring, rare, or conspicuous events capture our attention despite being task-irrelevant, that is, we get distracted. Distraction forces us to attend events which are beyond the focus of our task, which leads to decreased task-performance. But it also allows us to consider the wider context, and re-evaluate whether the task at hand is the most important thing to do at that moment or not. Reading the morning paper is important, figuring out why the alarm is blaring from the kitchen might be even more important. It seems plausible to assume that normal functioning is characterized by a balance between the ability to focus on a task, and being prone to distraction by potentially important sensory events. The goal of the present study was to explore our dynamic control over this balance.

One paradigm that has been successfully used to investigate distraction is the paradigm introduced by Schröger \& Wolff (1998b). In this paradigm, a series of tones are presented, and the participant's task is to respond to them according to one of their features (e.g. duration). The task-relevant feature-variants (e.g. short and long) are presented often (50$50 \%$ of the time, in random order). On most trials, the irrelevant stimulus-features are constant (standard trials), but randomly, from time-to-time, one irrelevant feature (e.g. pitch) is varied (independently from the task-relevant feature - these are called deviant trials).

Because participants perform the same discrimination task for deviants as for standards, differences in behavioral and ERP responses to deviants and standards are assumed to reflect distraction-related processing: Deviants elicit characteristic ERP waveforms in comparison to standards (Escera \& Corral, 2003): after deviance onset, a negativity between 80-200 ms 
comprising an N1-effect (Näätänen \& Picton, 1987), mismatch negativity (MMN, Näätänen, Gaillard, Matysalo, 1978), and possibly N2b (Näätänen, Simson, Loveless, 1982; Ritter et al., 1992) is observable, followed by the P3a (Friedman, Cycowicz, Gaeta, 2001) and the socalled reorienting negativity (RON, Schröger \& Wolff, 1998a). The same pattern of results was found in a similar arrangement with auditory distracters and visual task-relevant stimuli (e.g. Escera et al., 1998; Escera, Yago, Alho, 2001; Yago, Corral, Escera, 2001). These ERPs are usually interpreted in terms of three distraction-related processing stages. According to this model, the early negativities reflect processing in a sensory filter which highlights potentially significant stimulus events (first stage), P3a reflects the mobilization of attentional resources (second stage), and RON reflects the restoration of the task-optimal attention set (third stage) when the distracter needs no further action (for a recent summary, see Escera $\&$ Corral, 2007; also Horváth, Winkler, Bendixen, 2008). Whereas initial studies also showed that discrimination performance was lower and reaction times were delayed in deviant in comparison to standard trials (Schröger \& Wolff, 1998b; Schröger, Giard, Wolff, 2000), recent studies suggest that reaction times sum a number of effects unrelated to distraction per se (e.g. when the task does not engage participants sufficiently, distracters may substantially increase the level of arousal, or may allow participants to prepare more efficiently for the forthcoming task-relevant event), which may even result in performance enhancement in certain paradigms (Parmentier, Elsley, Ljungberg, 2010; SanMiguel et al., 2010a, 2010b; Wetzel, Widmann, Schröger, 2012; Ljungberg, Parmentier, Leiva, \& Vega, 2012; Li, Parmentier, \& Zhang, 2013; Wetzel, Schröger, \& Widmann, in press).A series of studies administering variations of the auditory distraction paradigm showed that visual cues signaling forthcoming, potentially distracting auditory events lead to the reduction of behavioral and event-related potential (ERP) correlates of distraction (Sussman, Winkler, Schröger, 2003; Wetzel \& Schröger, 2007; Wetzel, Widmann, \& Schröger, 2009; Horváth, 
Sussman, Winkler, Schröger, 2011; Horváth \& Bendixen, 2012). Although the results are compatible with the interpretation that distraction is prevented because participants actively counteract distraction on the basis of information provided by the cues, other explanations are also possible. One may argue that visual cue-processing may directly or indirectly tap into the resources utilized by distraction-related processes, and the apparent reduction of distraction is brought about by a "well-timed" processing interference, and not by targeted distractionprevention measures based on available information. That is, shortly after cue presentation, we might not momentarily have the processing resources to get distracted. Whereas some of the hypotheses regarding potential interference effects can be rejected (e.g., the assumption that deviant visual cues distract participants, and the "already distracted" participants cannot be "further distracted" by incoming auditory deviants can be rejected on the basis of Horváth et al., 2011), only a single behavioral study addressed the question whether the distractionprevention effect can be accounted for by increased processing-load due to voluntary cueprocessing. Parmentier and Hebrero (in press) hypothesized that cue processing overlapped, and might have interfered with distraction-related processing only when cues were presented shortly before the distracters. Because distraction-related reaction time delays were similarly reduced for short $(250 \mathrm{~ms})$ and long $(2250 \mathrm{~ms})$ cue-distracter separations, it seems likely that the preventive effect is based on the exploitation of cue information and not interference. The goal of the present study was to explore how the preparation interval influenced distractionprevention efficiency as reflected by the ERP correlates of distraction. The ERP-studies referenced above used preparation (cue-distracter) intervals ranging from 340 to $900 \mathrm{~ms}$, but each used only one interval. In the present study two preparation intervals were used. Because extracting and acting upon cue information takes time, it was hypothesized that the longer cue-distracter interval may allow for a more complete decoding of cue information and 
therefore a more efficient preparation than the short cue-distracter interval did, which would result in stronger reduction of behavioral and ERP distraction-effects. .

\section{Methods}

\subsection{Participants.}

21 young adults (age: 18-26 years, mean 22 years; 19 right, 2 left handed; 10 women) reporting normal hearing, and normal or corrected-to-normal vision, recruited through a student part-time job agency, participated in the experiment for monetary compensation. They give written informed consent before the experiment, after the experimental procedures were explained to them. Data was obtained from five further participants, but not used in the analyses: for four of these, the initial data processing after the first session showed an exceeding number of artifacts, so a second session was not administered; for the last of these five participants, the data was not used because after the artifact rejection procedures (see below) only half as many electroencephalogram (EEG) epochs as for the other participants remained.

\subsection{Stimuli and procedures.}

Participants were sitting in a comfortable armchair in a sound attenuated, dimly lit room during the experiment. Trials were presented with an onset-to-onset inter-trial interval of $1500 \mathrm{~ms}$. Trial structure is presented in Fig. 1. In each trial a tone was presented through HD-600 (Sennheiser, Wedemark, Germany) headphones. The tones were 100 or $200 \mathrm{~ms}$ long sinusoids (including 5-5 ms linear rise and fall times) of 988 or $1397 \mathrm{~Hz}$ frequency. Tone intensity was set individually to $50 \mathrm{~dB}$ sensation level (above hearing threshold level). The participants' task was to press a button held in their dominant hand for the long tones (irrespective of their frequency), but withhold response for short ones (irrespective of their frequency) (a Go/NoGo task). The instruction emphasized speeded responding, and after each 
experimental block feedback on reaction time distributions, mean response times and proportion of correct responses were presented and discussed. Short and long tone variations were presented in equal numbers (50-50\%) across trial types (see below). Tone frequencies were, however, asymmetric: one tone frequency was presented in $86.7 \%$ of the trials (standards), the other in $13.3 \%$ of the trials (deviants) in each experimental block. The roles of the tones were exchanged in different blocks.

A gray $\left(33 \mathrm{~cd} / \mathrm{m}^{2}\right), 0.45 \times 45^{\circ}$ (width $\times$ height) fixation cross was presented continuously on a black $\left(2 \mathrm{~cd} / \mathrm{m}^{2}\right)$ background during the experiment on a monitor in front of the participant (125 cm distance). Participants were instructed to direct their gaze on this fixation cross. In addition to the fixation cross, each tone was preceded by two visual stimuli: The first was presented $663 \mathrm{~ms}$ before the tone, the second $346 \mathrm{~ms}$ before the tone (onset-toonset intervals). Their duration was $100 \mathrm{~ms}$. These stimuli featured $1.23 \times 1.23^{\circ}$ gray $(33$ $\mathrm{cd} / \mathrm{m} 2$ ) squares with their center $1.83^{\circ}$ above (high cue) or below (low cue) the center of the fixation cross.

The cues informed participants about the frequency of the forthcoming tone (high or low) with $80 \%$ validity. For example, high cues were randomly followed by high tones in $80 \%$ of the trials and by low tones in the other $20 \%$. The two consecutive visual cues allowed the manipulation of cue information timing. In the long Cue-Tone Interval condition, the two visual stimuli were always the same (high or low) cues. In the short Cue-Tone Interval condition, the first stimulus featured both (high and low position) squares, and cue information became available only when the second visual stimulus was displayed. The presentation of two visual events was necessary to make sure that participants had the same fore-periods, and therefore the same opportunity to prepare for the presentation of the taskrelevant tone stimulus. 
The experiment consisted of two sessions which were separated by less than two weeks. At the beginning of the first session participants were familiarized with the task: First, one or two sequences of 60 tones (with random equiprobable selection from the four permutations of tone frequency and duration) were presented without visual stimuli to make sure that participants understood the task. To expose participants to the whole range of possible stimuli, the first 4 stimuli were always low-short, high-short, low-long and high-long tones.

Second, 3-4 sequences of 104 trials were presented with valid visual cues, short cuetone intervals. The tone frequency probabilities were asymmetric $(12.5 \%$ and $87.5 \%)$. The order of the first six trials was "standard, standard, deviant, standard, standard, deviant", in which the order of the first two trials were "short, long", in order to present the range of stimuli and demonstrate the cue-tone correspondence. Two similar blocks were administered at the beginning of the second session as a reminder to participants.

In the experimental phase, after mounting the electrodes, the independent variation of cue validity (valid or invalid) and tone (deviant or standard) resulted in 104 standards preceded by valid cues (valid-standard), 26 standards preceded by invalid cues (invalidstandard), 16 deviants preceded by valid cues (valid-deviant), and 4 deviants preceded by invalid cues (invalid-deviant) in each experimental block. In addition, the first six trials were "standard, standard, deviant, standard, standard, deviant" with valid cues, and the first two trials were "short, long", to present the range of stimuli and demonstrate the cue-tone correspondence. A total of 156 trials were presented in each block.

The independent variation of cue-tone interval and the (deviant or standard) role of tone pitch resulted in four types of blocks. Each type was presented 5 times in each session. Block order was randomized so that each block type was presented once in the 1-4, 5-8, 9-12, 
etc. block positions. Overall, 10 blocks of each block type was presented in two sessions. The blocks were separated by 1-2 minute breaks as needed, with a longer (10 minute) break after the $10^{\text {th }}$ block in each session.

\subsection{Analysis of the behavioral data}

Reaction times were analyzed in repeated measures three-way analysis of variance (ANOVA) with Cue-Tone Interval (short, long), Tone (standard, deviant), and Cue Validity (valid, invalid) factors. Only responses in the 150-800 ms time interval following the onset of long tones (correct responses) were included in the analysis. Similarly to the ERP analyses (see below), the first six tones in each block were excluded. Individual reaction times were calculated as the median of the reaction times. An ANOVA of the same structure was conducted for d' sensitivity scores calculated according to Signal Detection Theory (normal distribution model assuming equal variances; Macmillan and Creelman, 2005). Because in these calculations a higher number of trials may lead to an artificial bias in the corresponding d' scores, the numbers of hits, misses, correct rejections and false alarms were proportionally scaled down to match the lowest number of trials (the number of deviant trials preceded by an invalid cue), and rounded to the nearest integer. Hit rates of 1 and false alarm rates of zero were adjusted to $1-(1 /(2 \mathrm{~N}))$ and $1 /(2 \mathrm{~N})$, respectively (where $\mathrm{N}$ is the number of hits and correct rejections, respectively, see Macmillan and Creelman, 2005).

\subsection{EEG-recording and analysis.}

The EEG was recorded with a Synamp 2 (Compumedics Neuroscan, Victoria, Australia) amplifier with $500 \mathrm{~Hz}$ sampling rate, with on-line lowpass filtering (40Hz), using $\mathrm{Ag} / \mathrm{AgCl}$ electrodes mounted on an Easycap (Herrsching, Germany) elastic cap. 61 electrodes were positioned according to the $10 \%$ system (Nuwer, Comi, Emerson, Fuglsang-Frederiksen, Guérit, Hinrichs, et al., 1998). Further electrodes were placed at the mastoids. The reference 
electrode was placed at the tip of the nose, the ground on the forehead. The horizontal electrooculogram (EOG) was recorded with a bipolar setting with electrodes placed near the outer canthi of the eyes. A further electrode was placed under the right eye, which was used subtracted from the Fp2 signal to calculate the vertical EOG off-line.

The EEG was $20 \mathrm{~Hz}$ lowpass filtered (Kaiser-windowed sinc finite impulse response filter, beta of 5.65, 363 coefficients, $5 \mathrm{~Hz}$ transition bandwidth, with at least $60 \mathrm{~dB}$ stopband attenuation), and epochs of $1524 \mathrm{~ms}$, including a $770 \mathrm{~ms}$ pre-tone interval were extracted. Epochs with a signal range exceeding $150 \mu \mathrm{V}$ on any channel were discarded, as well as the epochs corresponding to the first six trials of each block. Individual average ERPs were calculated for all eight permutations of cue-tone interval (short or long), cue validity (valid or invalid) and tone (standard or deviant). Amplitude calculations were referred to the first 100 ms of the epochs.

The N1-effect/MMN/N2, P3a and RON were visually identified in the group-average "deviant after invalid cue-minus-standard after valid cue" difference waveforms for both long and short cue-tone intervals, because this difference should reflect the maximal level of distraction and the corresponding ERPs. The visual P3b was identified in the valid-deviantminus-valid-standard difference waveform (from the long Cue-Tone Interval condition, see Results section). Note that previous studies suggested (Horváth et al., 2011) that at least in the case of the short cue-tone interval the N1-effect/MMN/N2 might be the overlapped by the preceding visual P3b, therefore amplitude-effects in this interval should be interpreted with care.

The amplitudes of the N1-effect/MMN, P3a, and RON were measured at FCz; the N2 at $\mathrm{Cz}$; and the visual $\mathrm{P} 3 \mathrm{~b}$ at $\mathrm{Pz}$, because these sites were consistently reported as the sites of maximal amplitude in the literature (and the study by Horváth \& Bendixen, 2012, which used 
a highly similar settings). The individual amplitudes were calculated as the average signals in $40 \mathrm{~ms}$ long windows centered at the latency of the group-average difference waveform described above. The amplitudes were submitted to repeated measures three-way ANOVAs with Cue-Tone Interval (short, long), Tone (standard, deviant), and Cue Validity (valid, invalid) factors. The main interest in these analyses was whether a three-way interaction signaling that cue-tone interval modulated the effect of cue validity on the deviant-minusstandard difference was present.

For all ANOVAs generalized eta-squared effect sizes (Olejnik and Algina, 2003; Bakeman, 2005) are reported. The alpha-level was set to 0.05 . For all analyses all significant effects are reported.

\section{Results}

\subsection{Behavioral measures}

The group average reaction times results are shown in Fig. 2 (left). The results of the Cue-Tone Interval $\times$ Tone $\times$ Cue Validity ANOVA of the reaction times are shown in Table 1. To follow up the significant three-way interaction, separate Tone $\times$ Cue Validity ANOVAs were conducted, which (in addition to significant main effects, see below) revealed a significant interaction only in the long Cue-Tone Interval condition: $F(1,20)=14.19 ; \eta_{\mathrm{G}}{ }^{2}=$ $0.01, \mathrm{p}=0.001$. (In the long Cue-Tone Interval condition there was a Tone main effect $\mathrm{F}(1,20)=31.37 ; \eta_{\mathrm{G}}{ }^{2}=0.20, \mathrm{p}<0.001$; and a Cue Validity main effect: $\mathrm{F}(1,20)=35.29 ; \eta_{\mathrm{G}}{ }^{2}=$ $0.06, p<0.001$. In the short Cue-Tone condition there was a Tone main effect $F(1,20)=$ 33.10; $\eta_{\mathrm{G}}{ }^{2}=0.20, \mathrm{p}<0.001$; and a Cue Validity main effect: $\mathrm{F}(1,20)=6.91 ; \eta_{\mathrm{G}}{ }^{2}=0.004, \mathrm{p}=$ 0.02 . 
The group-average d' scores are shown in Fig. 2 (right). The Cue-Tone Interval $\times$ Tone $\times$ Cue Validity ANOVA showed a significant Tone main effect only: $F(1,20)=62.02$; $\eta_{\mathrm{G}}{ }^{2}=0.19, \mathrm{p}<0.001$, showing that sensitivity was lower in deviant than in standard trials.

\subsection{ERPs}

The group-average ERPs and the corresponding deviant-minus-standard waveforms are shown in Fig. 3 and 4, respectively. Following the transient ERP after the onset of the first visual stimulus, a slow negative trend is observable (Fig. 3) for both short and long cue-tone intervals. For the short cue tone-intervals (Fig. $3,1^{\text {st }}$ column) the deviant and standard ERPs show the same pattern till the elicitation of the tone-related N1. For long cue-tone intervals (Fig. $3,1^{\text {st }}$ column), the first visual stimulus leads to a bifurcation: deviant cues elicited a visual P3b in the -450 to $-200 \mathrm{~ms}$ interval (in reference to the tone onset). This was visually confirmed by the comparison of standards trials (Fig. $3,3^{\text {rd }}$ column), which shows that P3b was present only for deviant cues in the long cue-tone-interval condition but not in the others (obviously, the first visual stimulus is the same for deviant and standard cues for short cuetone intervals). Following the visual P3b, a slow negative shift, presumably a contingent negative variation (CNV, Walter et al., 1964; Tecce, 1972; Gaillard, 1976) was present for the stimuli in the long cue-tone interval condition in comparison to the short cue-tone interval condition (Fig.3, $3^{\text {rd }}$ and 4th columns). The significance of this unexpected finding was verified by a repeated measures Cue-Tone Interval $\times$ Tone $\times$ Cue Validity ANOVA of the average amplitudes measured in the $-100-0 \mathrm{~ms}$ interval at FCz (Table 2, Fig. 5).

As described in the Methods, distraction-related waveforms were identified in the “deviant after invalid cue-minus-standard after valid cue" difference waveforms for both long and short cue-tone intervals. The early negative difference (N1-effect/MMN/N2) showed two peaks, which are termed N1-effect and N2 in the following. The N1-effect peaked at $100 \mathrm{~ms}$ 
at Fpz in the short, and at $106 \mathrm{~ms}$ at FC4 in the long Cue-Tone Interval conditions. To accommodate both of these peaks, the window used for amplitude analysis was centered at $104 \mathrm{~ms}$. The more widely distributed N2 peaked at $176 \mathrm{~ms}$ at C2 in the short, and at $192 \mathrm{~ms}$ at PO8 in the long Cue-Tone Interval conditions. To accommodate both of these peaks, the amplitude analysis window was centered at $184 \mathrm{~ms}$. P3a peaked at $298 \mathrm{~ms}$ in the short, and at $300 \mathrm{~ms}$ in the long Cue-Tone Interval conditions at FCz. The amplitude analysis window was centered at $300 \mathrm{~ms}$. RON peaked at $400 \mathrm{~ms}$ in the short, and at $406 \mathrm{~ms}$ in the long Cue-Tone Interval conditions at FCz. The amplitude analysis window was centered at $404 \mathrm{~ms}$. The visual P3b was only observable in the long Cue-Tone Interval condition: it peaked at $-274 \mathrm{~ms}$ (i.e. $274 \mathrm{~ms}$ preceding tone-onset) at Pz. Fig. 4 shows that the topographical distributions of the ERPs corresponded well with that known from the literature.

The results of the amplitude analyses are presented in Table 2 and Fig. 5. There were only two ERPs for which a three-way interaction was found: the P3a and the visual P3b (note that for this calculation the time interval for the long cue-tone interval was used, because a P3b was not observed for the short cue-tone interval). Because the hypothesis is based on the presence of such interactions, these are discussed first.

To follow up the significant three-way interaction for the P3a interval, separate Tone $\times$ Cue Validity ANOVAs were conducted. For the short cue-tone interval a significant Tone main effect was found: $F(1,20)=20.14 ; \eta_{\mathrm{G}}{ }^{2}=0.159, \mathrm{p}<0.001$. For the long cue-tone interval, however, a significant Tone main effect: $F(1,20)=27.39 ; \eta_{\mathrm{G}}{ }^{2}=0.159, \mathrm{p}<0.001$; a Cue Validity main effect: $\mathrm{F}(1,20)=15.38 ; \eta_{\mathrm{G}}{ }^{2}=0.025, \mathrm{p}<0.001$; and significant Tone $\times$ Cue Validity interaction was found: $F(1,20)=5.48 ; \eta_{\mathrm{G}}{ }^{2}=0.005, \mathrm{p}=0.030$. This indicates that valid cues led to decreased P3a amplitudes, but only in the long cue-tone condition. 
To follow up the significant three-way interaction for the amplitudes in the visual P3b interval, separate Tone $\times$ Cue Validity ANOVAs were conducted. For the short Cue-Tone Interval condition no significant effects were found. For the long Cue-Tone Interval condition, however, a significant Cue Validity main effect: $F(1,20)=7.09 ; \eta_{\mathrm{G}}{ }^{2}=0.009, \mathrm{p}=$ 0.015 ; and significant Tone $\times$ Cue Validity interaction was found: $F(1,20)=70.56 ; \eta_{\mathrm{G}}{ }^{2}=$ $0.246, \mathrm{p}<0.001$. Obviously, the interaction stems from the fact that an invalid cue for a deviant is a cue signaling a forthcoming standard, and an invalid cue for a standard is a cue signaling a deviant.

In the N1-effect interval, a superposition of two (main) effects was found: one showed that deviants elicited larger N1s than standards, the other shows that the amplitudes were more negative for long-cut-tone intervals. This latter effect reflects the negative shift already observable before the tone onset, which is signaled by the main effect on the amplitudes measured in the $-100-0 \mathrm{~ms}$ interval (slow shift), and also in the $\mathrm{N} 2$ interval.

As expected, deviants resulted in more negative ERP amplitudes in the RON interval than standards. The Cue-Tone Interval $\times$ Cue Validity interaction was followed up by separate one-way Cue Validity ANOVAs. A significant effect was only found for long cue-tone intervals: $\mathrm{F}(1,20)=28.84 ; \eta_{\mathrm{G}}{ }^{2}=0.030, \mathrm{p}<0.001$, showing that invalid cues resulted in more negative amplitudes than valid cues in this time range.

\section{Discussion}

The results showed delayed reaction times and decreased duration-discrimination performance for deviants in comparison to standards. The deviant-minus-standard ERPs showed the succession of an N1-effect (possibly overlapped by an MMN), P3a and RON waveforms. These results are on-a-par with previous studies using similar experimental settings (e.g. Berti \& Schröger, 2001; Roeber, Berti, Schröger, 2003). Importantly, visual cues 
informing participants about forthcoming distracters reduced the distraction-related reaction time delay and P3a amplitude, but only when cues preceded tone-onset by $663 \mathrm{~ms}$. That is, the effects of distraction were reduced only when a longer preparation interval was available.

Whereas at first sight these results may seem to be compatible with the assumption that longer preparation intervals allow for a more efficient preparation, the fact that at a cuetone interval of $346 \mathrm{~ms}$ no modulation was observed, contradicts previous studies (Sussman et al., 2003; Horváth et al., 2011; Horváth \& Bendixen, 2012, Parmentier \& Hebrero, in press), which showed that it was possible to prevent distraction even when the preparation interval was similarly short. To shed more light on this discrepancy, the cue-related ERP results in the present study should be examined. Cues signaling a forthcoming deviant in the long CueTone Interval elicited a clear visual P3b, but no such P3b was observable in the short CueTone Interval condition, which suggests that participants did not utilize the cue information in the short Cue-Tone Interval condition (Johnson \& Donchin, 1978). This interpretation is corroborated by the presence of a slow negative shift (identifiable as a CNV, Walter et al., 1964; see also Tecce, 1972; Gaillard, 1976) following visual cues, which was higher (more negative) in the long Cue-Tone Interval condition, suggesting a stronger preparatory activity for the task-relevant sensory event. This suggests that although information on forthcoming tone distracters was available, participants were not using it; that is, they were not engaged in distraction preventing activities in the short Cue-Tone Interval condition, even though they could have prevented distraction.

It seems unlikely that cue information was not utilized because of a sensory limitation (e.g., visual masking): $317 \mathrm{~ms}$ between the uninformative first and the informative second visual stimulus should leave cue information easily perceivable in the short Cue-Tone Interval condition. Nonetheless the difference of the successive visual stimuli, combined with the shorter preparatory interval may render the distraction prevention task more difficult in the 
short than in the long Cue-Tone Interval condition. Therefore, the simplest explanation for this pattern of results is that participants made an effort to prevent distraction when cue information was available for a longer time (in a less complex stimulus sequence), but did not make such an effort when the cue information became accessible only shortly before the tone (in a more complex stimulation sequence). In other words, in this situation it might not have been "cost-effective" to prepare for the deviant when cue-tone interval was short: the effort needed might have been too much for too little effect in the short Cue-Tone Interval condition. One may also speculate that initially, preventing distraction might have been less successful for short cue-tone intervals, therefore participants quickly gave up trying, whereas the success at the long cue-tone intervals kept them engaged in this effort. This hypothetical difference in engagement was possibly facilitated by having the two conditions in different blocks, and by having no on-line measure or feedback on distraction prevention performance. Also, because of the duration of the paradigm, the ERP reflections of the initial efforts to utilize cue information for short cue-tone intervals may be rendered invisible by the process of averaging. A different speculation is that deviant cues might have been less effective as visual deviants per se in the two conditions. That is, in the context of the visual stimulus sequence, deviant cues might have been less easy to detect in the short than in the long CueTone Interval condition, that is, in the long condition the "displacement" of the square might have been more conspicuous than the "addition of the second square" in the short Cue-Tone condition. Because detecting the less conspicuous visual deviants might require more effort, participants may tacitly opt not to make the effort to utilize the cues in the short Cue-Tone Interval condition, whereas cues were utilized in the long Cue-Tone Interval condition, as well as in the studies cited above.

Although RON was present in both conditions, in contrast with previous studies (Sussman, et al., 2003; Horváth et al., 2011) its amplitude (i.e. the deviant-minus-standard 
difference) was not modulated by cue validity. Invalid cues, however, resulted in more negative ERP amplitudes in comparison to valid cues in the RON time range. This difference is probably caused by the differences in experimental design: In previous studies tones were either in complete correspondence with cues in one block, or they were completely independent from them in another. Because independent cues are irrelevant, participants do not make an effort to process them in task-related terms, which is reflected by the lack of deviant cue-related P3b (Sussman et al, 2003; Wetzel \& Schröger, 2007; Wetzel et al., 2009; Horváth et al., 2011). That is, in these studies, in each block, there were essentially only two types of stimuli (deviants and standards with valid cues in one block; deviants and standards in the other), whereas in the present study and in that by Horváth \& Bendixen (2012) four different events occurred in each block (the permutations of cue validity and tone type). In the study by Horváth \& Bendixen (2012), it was found that in the RON time-range standards and deviants preceded by invalid cues, and deviants preceded by valid cues elicited more negative amplitudes than standards following a valid cue. In the long cue-tone condition of the present study, invalid cues resulted in more negative amplitudes than valid cues. Whereas these two results do not perfectly match, both show that cue information affected RON amplitude. This suggests that the processes reflected by RON respond to events which are infrequent or unexpected in terms of the complex cue-tone events. In a certain sense RON seems to reflect processes which have access to the widest contextual information: The early N1-effect/MMN seems to be unaffected by cue information (Wetzel \& Schröger, 2007; Wetzel et al., 2009; Horváth et al., 2011); cue information affects P3a for deviants but not for standards; and RON seems to be affected by cue information and its relation to standards and deviants as well. Although some behavioral studies show differences between the processing of expected and unexpected standards (Parmentier et al., 2011), at this time the relationship between RON elicitation and such behavioral differences is difficult to assess. 
In summary, although the present study found a difference in cue utilization which fits the hypothesis that longer cue-tone intervals allow better preparation and more efficient distraction prevention, the lack of visual cue-related P3b in the short cue-tone condition, and a higher $\mathrm{CNV}$ following cues in the long cue-tone condition suggest that this was brought about by participants not making an effort to prevent distraction in the short cue-tone interval condition. Although this result is somewhat anticlimactic, it also shows that providing information about forthcoming distracters is not sufficient to prevent distraction, but the voluntary engagement of the participant is also required, which may vary between conditions. It is important to emphasize that assessing the participants' engagement in the prevention effort is not trivial. Whereas in the present study there was an obvious between-condition difference in visual P3b elicitation, as well as in the amplitude of the CNV emerging in the cue-tone interval, which suggested a difference in cue-related information processing and preparatory efforts, in similar studies the goal is to keep the level of engagement equal across conditions. Because the lack of statistically significant difference in these the cue-related P3b and CNV amplitudes does not guarantee equality in effort, future studies need to incorporate guarantees for the equality of engagement levels in the experimental designs. 


\section{Acknowledgements}

This research was supported by the European Community's Seventh Framework Programme (under grant agreement PERG04-GA-2008-239393), and the János Bolyai Research Scholarship of the Hungarian Academy of Sciences. I thank Zsuzsanna D’Albini for collecting the data. I also thank two anonymous reviewers for constructive comments. 


\section{References}

Bakeman R (2005) Recommended effect size statistics for repeated measures designs. Behavior Research Methods 37:379-384. doi: 10.3758/BF03192707

Berti S, Schröger E (2001) A comparison of auditory and visual distraction effects: behavioral and event-related indices. Cognitive Brain Research 10:265-273. doi: 10.1016/S09266410(00)00044-6

Escera C, Alho K, Winkler I, Näätänen R (1998) Neural mechanisms of involuntary attention to acoustic novelty and change. Journal of Cognitive Neuroscience 10:590-604. doi: $10.1162 / 089892998562997$

Escera C, Corral MJ (2007) Role of mismatch negativity and novelty-P3 in involuntary auditory attention. Journal of Psychophysiology 21:251-264. doi: 10.1027/02698803.21 .34 .251

Escera C, Corral M-J (2003) The distraction potential (DP), an electrophysiological tracer of involuntary attention control and its dysfunction. In: Reinvang I, Greenlee MW, Herrmann, M. (eds) The Cognitive Neuroscience of Individual Differences-New Perspectives. Bibliotheks-und Informationssystem der Universität Oldenburg, Oldenburg, Germany, pp 63-76

Escera C, Yago E, Alho K (2001) Electrical responses reveal the temporal dynamics of brain events during involuntary attention switching. European Journal of Neuroscience 14:877-883. doi: 10.1046/j.0953-816x.2001.01707.x

Friedman D, Cycowicz YM, Gaeta H (2001) The novelty P3: an event-related brain potential (ERP) sign of the brain's evaluation of novelty. Neuroscience and Biobehavioral Reviews 25:355-373. doi: 10.1016/S0149-7634(01)00019-7 
Gaillard AWK (1976) Effects of warning-signal modality on the contingent negative variation (CNV). Biological Psychology 4:139-153. doi: 10.1016/0301-0511(76)90013-2

Horváth J, Bendixen A (2012) Preventing distraction by probabilistic cueing. International Journal of Psychophysiology 83:342-347. doi: 10.1016/j.ijpsycho.2011.11.019

Horváth J, Sussman E, Winkler I, Schröger E (2011) Preventing distraction: Assessing stimulus-specific and general effects of the predictive cueing of deviant auditory events. Biological Psychology 87:35-48. doi: 10.1016/j.biopsycho.2011.01.011

Horváth J, Winkler I, Bendixen A (2008) Do N1/MMN, P3a, and RON form a strongly coupled chain reflecting the three stages of auditory distraction? Biological Psychology 79:139-147. doi: 10.1016/j.biopsycho.2008.04.001

Johnson R, Donchin E (1978) On how P300 amplitude varies with the utility of the eliciting stimuli. Electroencephalography and Clinical Neurophysiology 44:424-437. doi: 10.1016/0013-4694(78)90027-5

Li B, Parmentier FBR, Zhang M (2013) Behavioral distraction by auditory deviance is mediated by the sound's informational value. Experimental Psychology (formerly Zeitschrift für Experimentelle Psychologie) 60:260-268. doi: 10.1027/1618$3169 / \mathrm{a} 000196$

Ljungberg JK, Parmentier FBR, Leiva A, Vega N (2012) The informational constraints of behavioral distraction by unexpected sounds: The role of event information. Journal of Experimental Psychology: Learning, Memory, and Cognition 38:1461-1468. doi: $10.1037 / \mathrm{a} 0028149$

Macmillan NA, Creelman CD (2005) Detection theory: a user's guide, 2nd ed. Lawrence Erlbaum Associates, Mahwah, N.J 
Näätänen R, Gaillard AW, Mäntysalo S (1978) Early selective-attention effect on evoked potential reinterpreted. Acta Psychologica 42:313-329. doi: 10.1016/00016918(78)90006-9

Näätänen R, Picton T (1987) The N1 wave of the human electric and magnetic response to sound: A review and an analysis of the component structure. Psychophysiology 24:375425. doi: 10.1111/j.1469-8986.1987.tb00311.x

Näätänen R, Simpson M, Loveless NE (1982) Stimulus deviance and evoked potentials. Biological Psychology 14:53-98. doi: 10.1016/0301-0511(82)90017-5

Nuwer MR, Comi G, Emerson R, et al. (1998) IFCN standards for digital recording of clinical EEG. Electroencephalography and Clinical Neurophysiology 106:259-261. doi: 10.1016/S0013-4694(97)00106-5

Olejnik S, Algina J (2003) Generalized eta and omega squared statistics: Measures of effect size for some common research designs. Psychological Methods 8:434-447. doi: 10.1037/1082-989X.8.4.434

Parmentier FBR, Elsley JV, Andrés P, Barceló F (2011) Why are auditory novels distracting? Contrasting the roles of novelty, violation of expectation and stimulus change. Cognition 119:374-380. doi: 10.1016/j.cognition.2011.02.001

Parmentier FBR, Elsley JV, Ljungberg JK (2010) Behavioral distraction by auditory novelty is not only about novelty: The role of the distracter's informational value. Cognition 115:504-511. doi: 10.1016/j.cognition.2010.03.002

Parmentier FBR, Hebrero M (in press) Cognitive Control of Involuntary Distraction by Deviant Sounds. Journal of Experimental Psychology: Learning, Memory, and Cognition. doi: 10.1037/a0032421 
Ritter W, Paavilainen P, Lavikainen J, et al. (1992) Event-related potentials to repetition and change of auditory stimuli. Electroencephalography and Clinical Neurophysiology 83:306-321. doi: 10.1016/0013-4694(92)90090-5

Roeber U, Berti S, Schröger E (2003) Auditory distraction with different presentation rates: An event-related potential and behavioral study. Clinical Neurophysiology 114:341349. doi: $10.1016 / \mathrm{S} 1388-2457(02) 00377-2$

SanMiguel I, Linden D, Escera C (2010a) Attention capture by novel sounds: Distraction versus facilitation. European Journal of Cognitive Psychology 22:481-515. doi: $10.1080 / 09541440902930994$

SanMiguel I, Morgan HM, Klein C, et al. (2010b) On the functional significance of NoveltyP3: Facilitation by unexpected novel sounds. Biological Psychology 83:143-152. doi: 10.1016/j.biopsycho.2009.11.012

Schröger E, Giard M-H, Wolff C (2000) Auditory distraction: event-related potential and behavioral indices. Clinical Neurophysiology 111:1450-1460.

Schröger E, Wolff C (1998a) Attentional orienting and reorienting is indicated by human event-related brain potentials. Neuroreport 9:3355-3358.

Schröger E, Wolff C (1998b) Behavioral and electrophysiological effects of task-irrelevant sound change: A new distraction paradigm. Cognitive Brain Research 7:71-87. doi: 10.1016/S0926-6410(98)00013-5

Sussman E, Winkler I, Schröger E (2003) Top-down control over involuntary attention switching in the auditory modality. Psychonomic Bulletin \& Review 10:630-637. doi: 10.3758/BF03196525 
Tecce JJ (1972) Contingent negative variation (CNV) and psychological processes in man. Psychological Bulletin 77:73-108. doi: 10.1037/h0032177

Walter WG, Cooper R, Crow HJ, et al. (1967) Contingent negative variation and evoked responses recorded by radio-telemetry in free-ranging subjects. Electroencephalography and Clinical Neurophysiology 23:197-206. doi: 10.1016/0013-4694(67)90116-2

Wetzel N, Schröger E (2007) Cognitive control of involuntary attention and distraction in children and adolescents. Brain Research 1155:134-146. doi:

10.1016/j.brainres.2007.04.022

Wetzel N, Schröger E, Widmann A (in press) The dissociation between the P3a event-related potential and behavioral distraction. Psychophysiology n/a-n/a. doi:

10.1111/psyp. 12072

Wetzel N, Widmann A, Schröger E (2009) The cognitive control of distraction by novelty in children aged 7-8 and adults. Psychophysiology 46:607-616. doi: 10.1111/j.14698986.2009.00789.x

Wetzel N, Widmann A, Schröger E (2012) Distraction and facilitation-two faces of the same coin? Journal of Experimental Psychology: Human Perception and Performance 38:664-674. doi: 10.1037/a0025856

Yago E, Corral MJ, Escera C (2001) Activation of brain mechanisms of attention switching as a function of auditory frequency change. Neuroreport 12:4093-4097. 
Tables

\begin{tabular}{|l|r|r|r|}
\hline Effect & $\mathbf{F ( 1 , 2 0 )}$ & $\mathbf{p}$ & $\mathbf{\eta}_{\mathbf{G}}{ }^{2}$ \\
\hline Tone & 33.95 & $<0.001$ & 0.199 \\
\hline Cue Validity & 31.30 & $<0.001$ & 0.023 \\
\hline Cue-Tone Interval $\times$ Cue Validity & 18.78 & $<0.001$ & 0.007 \\
\hline Tone $\times$ Cue Validity & 4.67 & 0.043 & 0.002 \\
\hline Cue-Tone Interval $\times$ Tone $\times$ Cue Validity & 5.37 & 0.031 & 0.002 \\
\hline
\end{tabular}

Table 1. Significant effects in the ANOVA of the reaction times.

\begin{tabular}{|c|c|c|c|c|}
\hline $\begin{array}{l}\text { ERP } \\
\text { (interval) }\end{array}$ & Effect & $\mathbf{F}(\mathbf{1 , 2 0})$ & $\mathbf{p}$ & $\eta_{G^{2}}^{2}$ \\
\hline \multirow{2}{*}{$\begin{array}{l}\text { N1-eff. } \\
(84-124 \mathrm{~ms})\end{array}$} & Cue-Tone Interval & 34.08 & $<0.001$ & 0.046 \\
\hline & Tone & 27.20 & $<0.001$ & 0.013 \\
\hline $\begin{array}{l}\mathrm{N} 2 \\
(164-204 \mathrm{~ms})\end{array}$ & Cue-Tone Interval & 21.33 & $<0.001$ & 0.019 \\
\hline \multirow{4}{*}{$\begin{array}{l}\text { P3a } \\
(280-320 \mathrm{~ms})\end{array}$} & Tone & 23.89 & $<0.001$ & 0.159 \\
\hline & Cue Validity & 9.37 & 0.006 & 0.008 \\
\hline & Cue-Tone Interval $\times$ Cue Validity & 8.12 & 0.010 & 0.004 \\
\hline & Cue-Tone Interval $\times$ Tone $\times$ Cue Validity & 5.44 & 0.030 & 0.002 \\
\hline $\mathrm{RON}$ & Tone & 27.58 & $<0.001$ & 0.072 \\
\hline$(384-424 \mathrm{~ms})$ & Cue Validity & 7.08 & 0.015 & 0.010 \\
\hline
\end{tabular}




\begin{tabular}{|l|l|r|r|c|}
\hline & Cue-Tone Interval $\times$ Cue Validity & 8.95 & 0.007 & 0.004 \\
\hline \multirow{3}{*}{$(-294--254 \mathrm{~ms})$} & & & & \\
\cline { 2 - 5 } & Cue Validity & 28.79 & $<0.001$ & 0.088 \\
\cline { 2 - 5 } & Tone $\times$ Cue Validity & 4.60 & 0.044 & 0.005 \\
\cline { 2 - 5 } & Cue-Tone Interval $\times$ Tone $\times$ Cue Validity & 34.93 & $<0.001$ & 0.072 \\
\hline \multirow{2}{*}{ Slow negative shift } & Cue-Tone Interval & 52.14 & $<0.001$ & 0.091 \\
\hline (-100-0 ms) & & 23.79 & $<0.001$ & 0.059 \\
\hline
\end{tabular}

Table 2. Significant effects in the Cue-Tone Interval $\times$ Tone $\times$ Cue Validity ANOVAs of the ERP amplitudes Each ERP amplitude was calculated as the average signal in the given timewindows). 


\section{Figure captions}

Fig. 1 Trial structure. Stimulus timings are represented by black bars above the ruler. The duration of visual stimuli was $100 \mathrm{~ms}$, tones were 100 or $200 \mathrm{~ms}$ long. Cues were squares presented above or below the fixation cross. On valid trials, cue position (above or below the fixation cross) corresponded to tone frequency (high or low). In the long Cue-Tone Interval condition, the first and the second visual stimuli are the same informative cues. In the short Cue-Tone Interval condition both cues are presented as the first visual stimulus, but only one of the cues as the second.

Fig 2. Group-average $(\mathrm{N}=21)$ reaction time (referred to tone onset time) and d' sensitivity scores in the experiment.

Fig 3. Group-average $(\mathrm{N}=21)$ ERP waveforms elicited by deviants and standards preceded by valid or invalid cues in the short $\left(1^{\text {st }}\right.$ column $)$ and long $\left(2^{\text {nd }}\right.$ column $)$ cue-tone interval conditions at the Fz, FCz, Cz, CPz, Pz leads and the average of the mastoids $(\mathrm{CM})$. The $3^{\text {rd }}$ column shows the same standard ERPs as in the $1^{\text {st }}$ and $2^{\text {nd }}$ columns, whereas the $4^{\text {th }}$ column shows the same deviant ERPs as in the $1^{\text {st }}$ and $2^{\text {nd }}$ columns. Arrows mark the visual P3b in the 2nd, 3rd, and $4^{\text {th }}$ columns at $\mathrm{Pz}$, and the slow shift following the onset of the second visual stimulus at FCz. Stimulus onset times are marked on the rulers by arrows, and by slashed vertical lines at the ERPs. Tone onset is at the crossing of the axes.

Fig. 4 Group-average $(\mathrm{N}=21)$ deviant-minus-standard difference waveforms in the short cuetone interval ( $1 \mathrm{st}$ and $2^{\text {nd }}$ columns) and the long cue-tone interval ( $3 \mathrm{rd}$ and 4 th columns) conditions at Fz, FCz, Cz, CPz, Pz leads and the average of the mastoids (CM). The $1^{\text {st }}$ and $3^{\text {rd }}$ columns show waveforms for ERP differences between trials with identical cues, that is, "deviant after invalid cue - minus - standard after valid cue", and "deviant after valid cue minus - standard after invalid cue" differences are presented. In the $2^{\text {nd }}$ and $4^{\text {th }}$ column, the 
cues differed, that is "deviant after valid cue - minus - standard after valid cue" and "deviant after invalid cue - minus - standard after invalid cue" differences are presented. Arrows indicate the N1-effect, N2, P3a and RON waveforms at FCz in the $3^{\text {rd }}$ column, and the visual $\mathrm{P} 3 \mathrm{~b}$ at $\mathrm{Pz}$ in the $4^{\text {th }}$ column. Stimulus onset times are marked on the rulers by arrows, and by slashed vertical lines at the ERPs. Tone onset is at the crossing of the axes. Note that vertical scaling differs from that of Fig. 3.

Fig. 5 Group-average $(\mathrm{N}=21)$ ERP amplitudes in the N1-effect, N2, P3a, RON, the visual P3b (assessed in the long cue-tone interval condition) time intervals, and the slow negative shift following the cues. For the N1-effect, P3a and RON, and the slow negative shift measurements were made at $\mathrm{FCz}$, for $\mathrm{N} 2$ at $\mathrm{Cz}$, and for the visual P3b at Pz. Negativity is upwards on the vertical scale to match Fig. 3 and 4. 


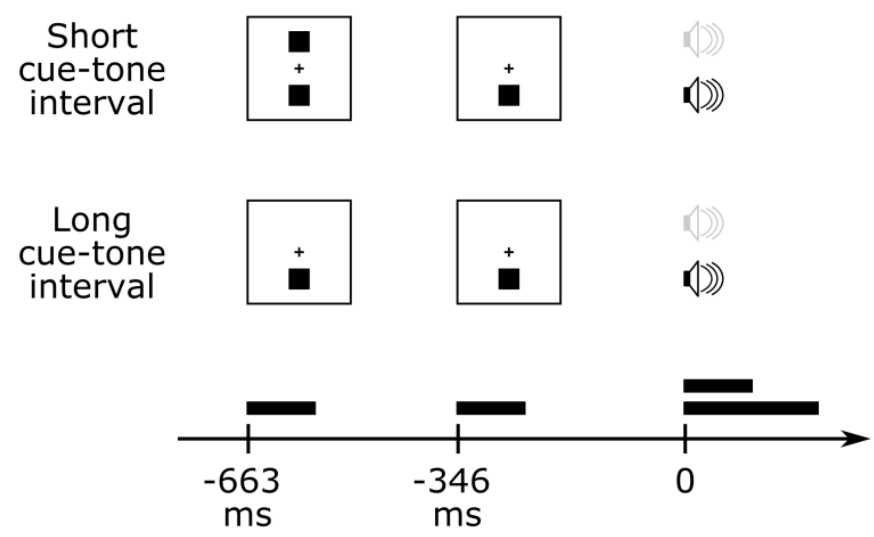

Fig. 1 Trial structure. Stimulus timings are represented by black bars above the ruler. The duration of visual stimuli was $100 \mathrm{~ms}$, tones were 100 or $200 \mathrm{~ms}$ long. Cues were squares presented above or below the fixation cross. On valid trials, cue position (above or below the fixation cross) corresponded to tone frequency (high or low). In the long Cue-Tone Interval condition, the first and the second visual stimuli are the same informative cues. In the short Cue-Tone Interval condition both cues are presented as the first visual stimulus, but only one of the cues as the second. 

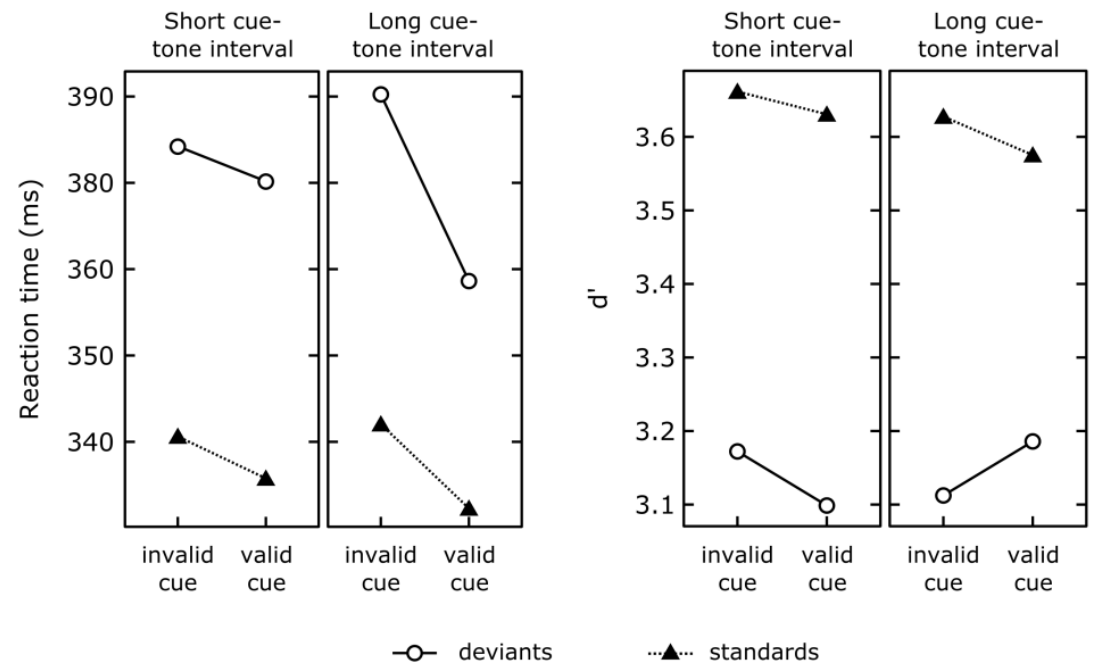

Fig 2. Group-average $(\mathrm{N}=21)$ reaction time (referred to tone onset time) and d' sensitivity scores in the experiment. 


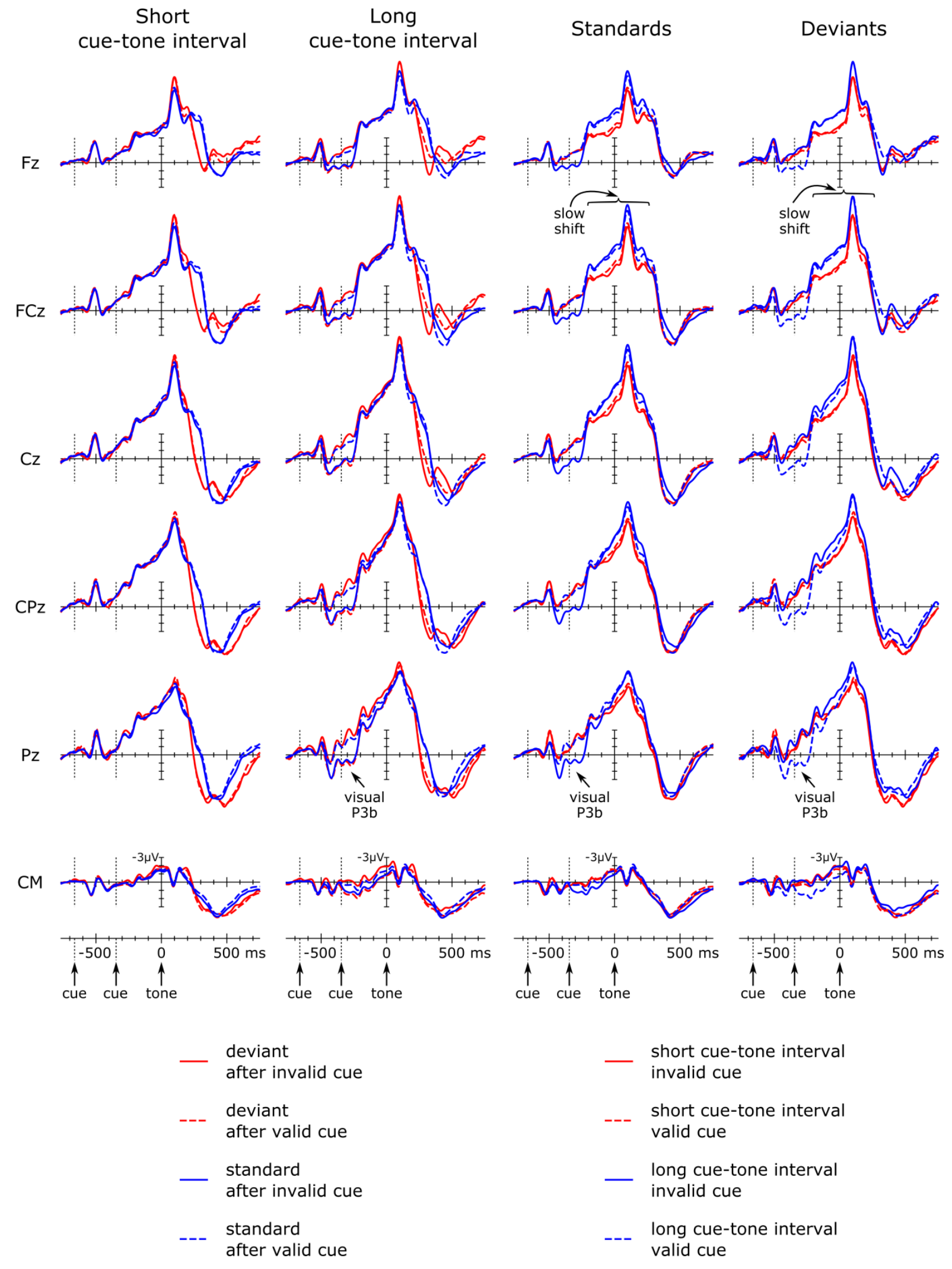

Fig 3. Group-average ( $\mathrm{N}=21)$ ERP waveforms elicited by deviants and standards preceded by valid or invalid cues in the short $\left(1^{\text {st }}\right.$ column $)$ and long $\left(2^{\text {nd }}\right.$ column $)$ cue-tone interval 
conditions at the Fz, FCz, Cz, CPz, Pz leads and the average of the mastoids $(\mathrm{CM})$. The $3^{\text {rd }}$ column shows the same standard ERPs as in the $1^{\text {st }}$ and $2^{\text {nd }}$ columns, whereas the $4^{\text {th }}$ column shows the same deviant ERPs as in the $1^{\text {st }}$ and $2^{\text {nd }}$ columns. Arrows mark the visual P3b in the 2nd, 3rd, and $4^{\text {th }}$ columns at $\mathrm{Pz}$, and the slow shift following the onset of the second visual stimulus at FCz. Stimulus onset times are marked on the rulers by arrows, and by slashed vertical lines at the ERPs. Tone onset is at the crossing of the axes. 
Deviant-minus-standard difference waveforms

Short cue-tone interval

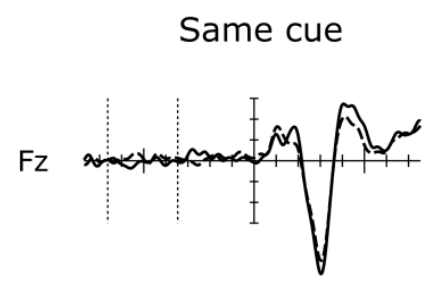

FCz

$\mathrm{Cz}$

$\mathrm{CPz}-1$

Pz

CM

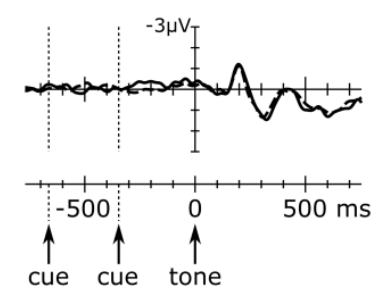

- deviant - invalid cue
minus

standard - valid cue

--- deviant - valid cue minus

standard - invalid cue
Different cue
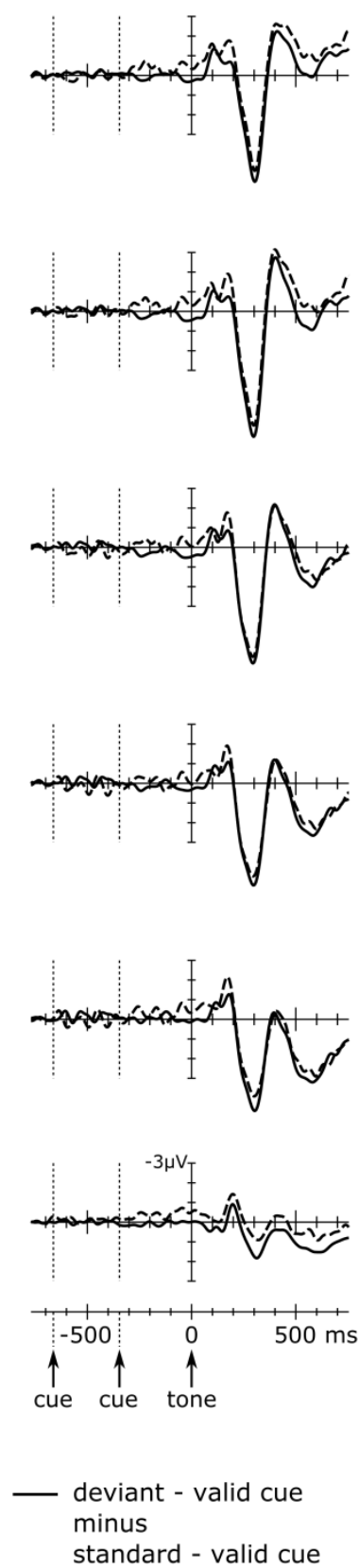
--- deviant - invalid cue minus standard - invalid cue

Long cue-tone interval

Same cue

Different cue
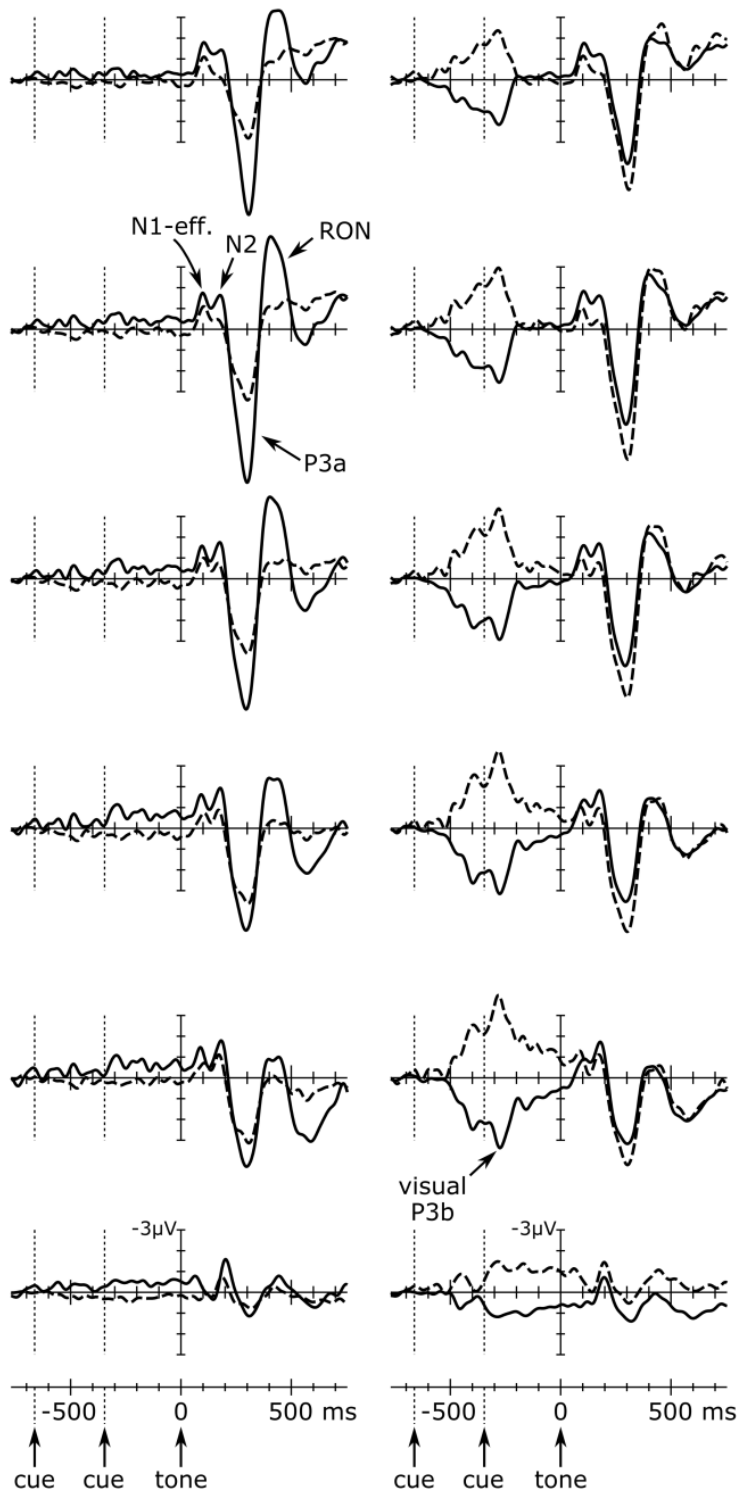

$$
\begin{array}{lr}
- \text { deviant - invalid cue } & \text { deviant - valid cue } \\
\text { minus } & \begin{array}{l}
\text { minus } \\
\text { standard - valid cue }
\end{array} \\
\text { standard - valid cue } & \\
\text {--- deviant - valid cue } & -- \text { deviant - invalid cue } \\
\text { minus } & \begin{array}{l}
\text { minus } \\
\text { standard - invalid cue }
\end{array}
\end{array}
$$

Fig. 4 Group-average ( $\mathrm{N}=21)$ deviant-minus-standard difference waveforms in the short cuetone interval ( $1 \mathrm{st}$ and $2^{\text {nd }}$ columns) and the long cue-tone interval ( $3 \mathrm{rd}$ and 4 th columns) conditions at $\mathrm{Fz}, \mathrm{FCz}, \mathrm{Cz}, \mathrm{CPz}, \mathrm{Pz}$ leads and the average of the mastoids (CM). The $1^{\text {st }}$ and $3^{\text {rd }}$ 
columns show waveforms for ERP differences between trials with identical cues, that is, "deviant after invalid cue - minus - standard after valid cue", and "deviant after valid cue minus - standard after invalid cue" differences are presented. In the $2^{\text {nd }}$ and $4^{\text {th }}$ column, the cues differed, that is "deviant after valid cue - minus - standard after valid cue" and "deviant after invalid cue - minus - standard after invalid cue" differences are presented. Arrows indicate the N1-effect, N2, P3a and RON waveforms at FCz in the $3^{\text {rd }}$ column, and the visual $\mathrm{P} 3 \mathrm{~b}$ at $\mathrm{Pz}$ in the $4^{\text {th }}$ column. Stimulus onset times are marked on the rulers by arrows, and by slashed vertical lines at the ERPs. Tone onset is at the crossing of the axes. Note that vertical scaling differs from that of Fig. 3. 


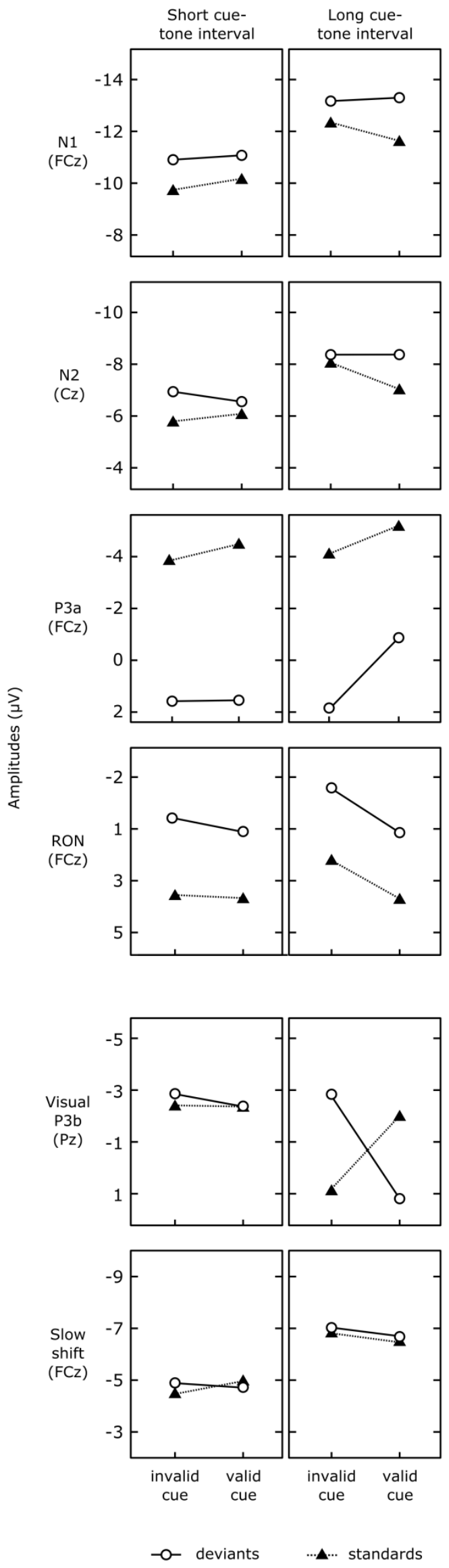


Fig. 5 Group-average (N=21) ERP amplitudes in the N1-effect, N2, P3a, RON, the visual P3b (assessed in the long cue-tone interval condition) time intervals, and the slow negative shift following the cues. For the N1-effect, P3a and RON, and the slow negative shift measurements were made at $\mathrm{FCz}$, for $\mathrm{N} 2$ at $\mathrm{Cz}$, and for the visual P3b at Pz. Negativity is upwards on the vertical scale to match Fig. 3 and 4. 